\title{
Ultrafast Radial Transport In A Micron-Scale Aluminum Plasma Excited At Relativistic Intensity
}

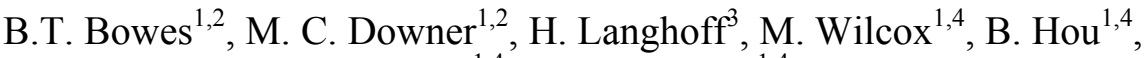 \\ J. Nees ${ }^{1,4}$ and G. Mourou ${ }^{1,4}$ \\ ${ }^{1}$ FOCUS Center \\ ${ }^{2}$ Department of Physics, University of Texas at Austin, Austin, Texas, USA 78712 \\ ${ }^{3}$ Physikalisches Institut der Universität Würzburg, 97074 Würzburg, Germany \\ ${ }^{4}$ Center for Ultrafast Optical Science, University of Michigan, Ann Arbor, Michigan, USA 48109
}

\begin{abstract}
Using femtosecond microscopy, we observe a thermal/ionization front expand radially at $\sim 10^{8} \mathrm{~cm} / \mathrm{s}$ from a $\lambda^{2}$-size spot of an aluminum target excited at $>10^{18} \mathrm{~W} / \mathrm{cm}^{2}$. Numerical modeling shows transport is predominantly radiative and may be initially nonlocal.
\end{abstract}

Excitation of solid targets with laser pulses of relativistic intensity has recently emerged as a promising method for producing collimated beams of $\mathrm{MeV}$ protons and ions $[1,2]$. In order to tailor the energy, energy spread and quantity of particles in a beam to a specific scientific query or engineering application the physics between initial laser energy deposition and emission of an ion beam must be fully understood. To this end, we present an experiment in which ultrafast energy deposition on any target material is optically probed to observe the dynamics of energy flow in the target with very high temporal and spatial resolution.

Intense, high contrast femtosecond (fs) laser pulses deposit energy into the electrons of a solid faster than it escapes from the initially-excited volume and much faster than the target surface expands hydrodynamically. When initial electron temperature $\mathrm{kT}_{\mathrm{e}}$ exceeds several hundred $\mathrm{eV}$ radiative heat transport begins to dominate over collisional transport [3]. The physics of this regime underlies production of $\mathrm{MeV}$ proton and ion beams, as well as energy transport in stars and ultrashort pulse x-ray generation. Past experiments in this regime [3] used loosely focused, $\sim 1 \mathrm{~J}, \sim 1 \mathrm{ps}$ pump pulses and probed the target transversely in transmission, and thus were restricted to observing late stages of $1 \mathrm{D}$ radiative transport in an optically transparent material on a time scale of tens of picoseconds. We present new measurements using $1 \mathrm{~mJ}, 24 \mathrm{fs}$ pump pulses focused to a diffraction-limited $\lambda^{2}$-size spot $(1.5 \mu \mathrm{m}$ diameter) to excite a metal target surface at relativistic intensity (up to 1.8 $\times 10^{18} \mathrm{~W} / \mathrm{cm}^{2}$ ). We probe the target in reflection through microscope optics. This geometry enables us to observe the earliest stages of radiative transport in 2D on any target material on a sub-picosecond time scale. New features of radiative transport are expected on this space-time scale. For example, simple transport models in the 
diffusive limit (i.e. Rosseland radiation mean free path $\lambda_{R}<<$ heated spot size $\lambda$ ) predict very different temporal evolution of the thermal/ionization front in $2 \mathrm{D}$ vs. $1 \mathrm{D}$ [5]. Moreover, since our focal spot size is on the order of $\lambda_{R}$, our experiment may open up fs studies of nonlocal radiative transport.

Pump pulses, focused with an $\mathrm{f} / 1$ off-axis paraboloid coupled to adaptive optics [4], excited the $\mathrm{Al}$ target at $1 \mathrm{kHz}$ repetition rate. At the highest focused pump intensity, $\mathrm{K} \alpha$ and continuum bremsstrahlung $\mathrm{x}$-rays are clearly observed (Fig. 1a, top), prima facie evidence that relativistic intensity and $\mathrm{keV}$ electron temperatures have been reached. The target is translated so that each laser shot interacts with clean target material. Surface wobble is limited to $<1 \mu \mathrm{m}$, resulting in highly reproducible damage spots (Fig. 1a, left-middle). The probe is split from the pump, frequency-doubled, and focused at $60^{\circ}$ incidence angle to a $\sim 10 \mu \mathrm{m}$ spot centered on the pump spot. The reflected probe is then imaged with high quality microscope optics onto a CCD camera, which records $140 \times$ magnified images of the pump-excited spot with $<1 \mu \mathrm{m}$ spatial resolution. The three selected images of a p-polarized probe in Fig. 1a (bottom) show a smooth Gaussian profile at $\Delta \mathrm{t}<0$. By $\Delta \mathrm{t}=0.18 \mathrm{ps}$, the pump-excited area has darkened. By $\Delta \mathrm{t} \approx 0.5 \mathrm{ps}$, the "dark" region has doubled in diameter, implying radial expansion at $\mathrm{v}_{\mathrm{rad}} \approx 10^{8} \mathrm{~cm} / \mathrm{s}$. Simultaneously, diffraction features caused by local surface curvature appear. The lineouts of the imaged probe in Fig. $1 \mathrm{~b}$ show the temporal evolution of the central dark region in more detail. At the highest pump intensity $\left(1.8 \times 10^{18} \mathrm{~W} / \mathrm{cm}^{2}\right)$, it expands well beyond the directly photo-excited area within 1 ps. At $5 \times$ lower pump intensity $\left(3.7 \times 10^{17} \mathrm{~W} / \mathrm{cm}^{2}\right)$, on the other hand, it remains confined largely within the pump-excited area.

The darkening in the center of the images indicates heating and ionization of the Al surface, but its physical origin must be understood to model transport quantitatively. To this end, Fig. 2a plots normalized change $\Delta R_{\text {probe }}(\Delta t) / R_{0}$ in $p$ - and s-polarized probe reflectivity for excitation at $1.8 \times 10^{18} \mathrm{~W} / \mathrm{cm}^{2}$ obtained from the measuring the center of the imaged pump-excited region. $\Delta \mathrm{R}$ is negligible for an s-polarized probe, demonstrating that the drop of the p-polarized reflectivity is caused by resonance absorption (RA) [6] at the expanding critical surface. The critical surface expands vertically at the ion acoustic velocity $\mathrm{v}_{\mathrm{z}} \approx\left(\mathrm{ZkT}_{\mathrm{e}} / \mathrm{m}_{\mathrm{i}}\right)^{1 / 2}$, and maximum RA occurs when it expands to $\mathrm{v}_{\mathrm{z}} \Delta \mathrm{t}_{\max } \approx \lambda_{\text {probe }} / 8 \approx 50 \mathrm{~nm}$. [8]. From Fig. $2 \mathrm{a}, \Delta \mathrm{t}_{\max } \approx 0.3$ ps, implying $\mathrm{v}_{\mathrm{z}}$ $\approx 2 \times 10^{7} \mathrm{~cm} / \mathrm{s}$. This $\mathrm{v}_{\mathrm{z}}$ is consistent with ionization state $\mathrm{Z} \approx 10$ and $\mathrm{kT}_{\mathrm{e}} \approx$ several $\mathrm{keV}$. The slight delay between ionizing and heating a point on the surface to $\left(\mathrm{Z}, \mathrm{kT}_{\mathrm{e}}\right)$ and the appearance of maximum RA must be taken into account in modeling the radial expansion data. However, since vertical expansion $\mathrm{v}_{\mathrm{z}} \Delta \mathrm{t}$ remains much less than the radial spot size during the time interval $(0<\Delta \mathrm{t}<1 \mathrm{ps})$ of interest, a 1 -D hydrodynamic model like MEDUSA [7] is sufficient for this purpose. Although pre-pulses at the $10^{-}$ ${ }^{5}$ level were present, no $\Delta \mathrm{R}_{\text {probe }}$ occurred before arrival of the main pump pulse (Fig. 2b).

We modeled the evolution of the electron temperature profile $\mathrm{T}_{\mathrm{e}}(\mathrm{r}, \mathrm{z} \leq 0, \mathrm{t})$ at constant solid density $\rho$ by numerically solving the nonlinear diffusion equation $\partial \mathrm{T}_{\mathrm{e}} / \partial \mathrm{t}$ $=\nabla \cdot\left(\chi \nabla \mathrm{T}_{\mathrm{e}}\right)$ with a temperature-dependent thermal diffusivity $\chi=\left(\kappa_{\mathrm{SH}}+\kappa_{\mathrm{R}}\right) / \rho \mathrm{c}_{\mathrm{v}}$ that included collisional (Spitzer-Härm) conductivity $\kappa_{\mathrm{SH}} \sim\left(\mathrm{kT}_{\mathrm{e}}\right)^{5 / 2} /(\mathrm{Z}+1)$ and radiative conductivity $\kappa_{\mathrm{R}}=16 \sigma \mathrm{T}_{\mathrm{e}}{ }^{3} \lambda_{\mathrm{R}} / 3$ [5], where $\sigma$ is the Stefan-Boltzman constant and 
$\lambda_{\mathrm{R}}[\mathrm{cm}]=\left(9 \times 10^{6}\right) \mathrm{T}_{\mathrm{e}}\left[{ }^{\mathrm{o}} \mathrm{K}\right]^{2} / \mathrm{Zn}_{\mathrm{e}}\left[\mathrm{cm}^{-3}\right]$ is a simplified radiative mean free path valid for hydrogenic ions $[3,5]$. The initial condition was defined by partitioning absorbed pump energy $(\sim 1 \mathrm{~mJ})$, with Gaussian radial profile, between electron thermal energy $\mathrm{kT}_{\mathrm{e}}$ and ionization $\mathrm{Z}\left(\mathrm{kT}_{\mathrm{e}}\right)$ assuming Saha equilibrium. The choice of initial absorption depth $Z_{a b s}$ was not critical because $T_{e}(r, z \leq 0, t)$ quickly evolved to a nearly hemispherical profile which became the effective initial condition.
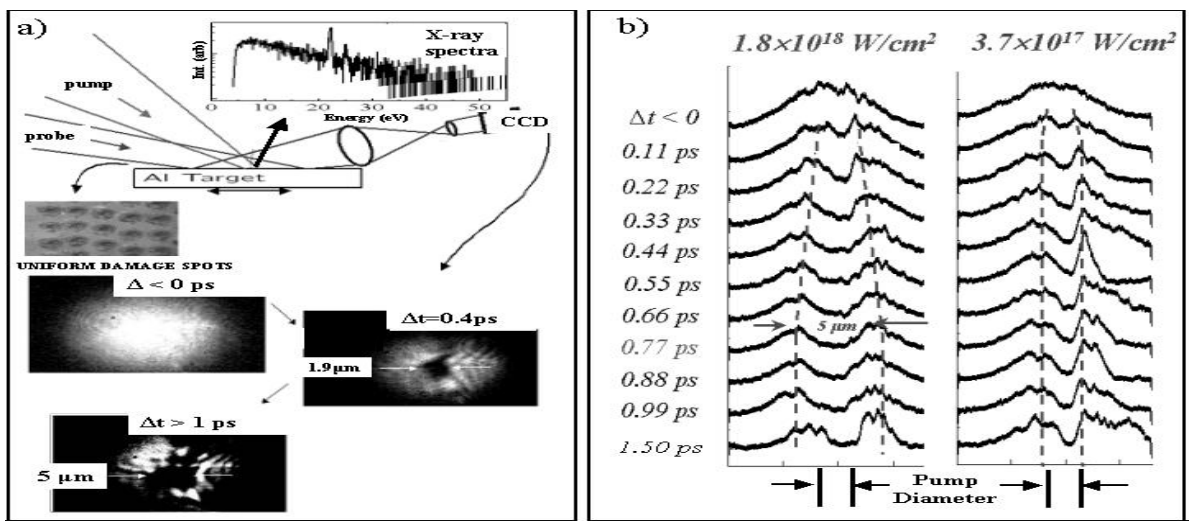

FIGURE 1. a) Diagram of experimental setup for optical and X-ray measurements and typical probe beam images. b) Lineouts from probe images at two different pump laser intensities. The large radial expansion of the laser excited region is clearly observed at the highest pump intensity (left) but is nearly absent when the pump laser intensity is reduced by a factor of approximately five to $3.7 \times 10^{17} \mathrm{~W} / \mathrm{cm}^{2}$.

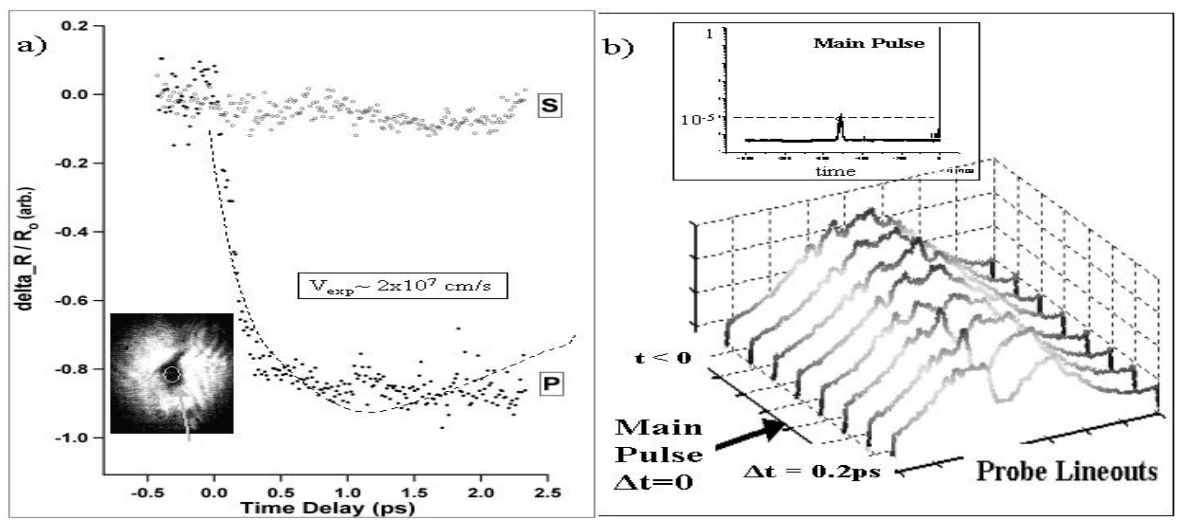

FIGURE 2. a) Normalized S- and P-polarized probe beam reflectivity $\Delta$ Rprobe $(\Delta t) / R 0$ of the center of the laser excited region for pump intensity $1.8 \times 1018 \mathrm{~W} / \mathrm{cm} 2$. Dashed curve: fit of p-polarized data to a 1-D resonance absorption model [6], assuming constant vertical expansion velocity $\mathrm{vz}=2 \times 107 \mathrm{~cm} / \mathrm{s}$. The data deviate from the model for $\Delta \mathrm{t}>1 \mathrm{ps}$ because the critical surface expansion becomes 3-D and kTe has cooled. b) Probe image lineouts before and after the arrival of the intense pump pulse. The reflectivity of the probe beam does not change until after the arrival of the pump pulse due to the high contrast of the pump laser pulse (inset). Pre-pulses at the 10-5 level are measured. 
We benchmarked the code by reproducing the $1 \mathrm{D}$ transport results of Ref. [3]. We then completed the model of our results by coupling the calculated $T_{e}(r, z<0, t)$ to the 1D hydrodynamic model assuming $T_{e}(r, z>0, t)=T_{e}(r, 0, t)$. This approximation is justified by the low heat capacity and high thermal conductivity of the expanding coronal plasma.

Fig. 3a shows the calculated profile of the critical surface $S(r, z)$ at several $\Delta t$ for excitation at $1.8 \times 10^{18} \mathrm{~W} / \mathrm{cm}^{2}$. During $0<\Delta \mathrm{t}<1 \mathrm{ps}$, S expands vertically at nearly constant $\mathrm{v}_{\mathrm{z}} \sim 2 \times 10^{7} \mathrm{~cm} / \mathrm{s}$. Simultaneously it expands radially, at first rapidly, then rapidly decelerating because of the highly nonlinear $\chi$. The radius $r_{c}$ of the "dark" spot was taken to be the radius of $\mathrm{S}(\mathrm{r}, \mathrm{z})$ at $\mathrm{z}=\lambda_{\text {probe }} / 8$, which corresponds to maximum RA. The resulting time evolution $r_{c}(\Delta t)$ is plotted in Fig. $3 b$ (solid curve) and compared with the measured HWHM $r_{\text {dark }}(\Delta t)$ of the dark spot (dark circles). A similar calculated curve for excitation at $3.7 \times 10^{17} \mathrm{~W} / \mathrm{cm}^{2}$ is compared with the corresponding data (triangles). Both curves reproduce the measured spatial extent of radial expansion quite well. For contrast, the calculated $r_{c}(\Delta t)$ for excitation at

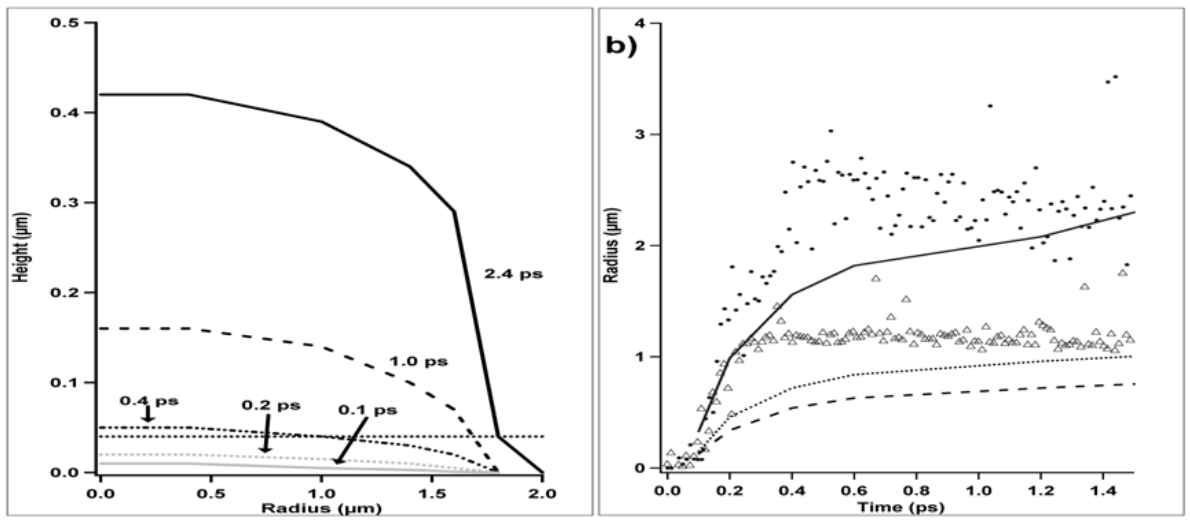

FIGURE 3. a) Calculations of expanding critical surface of the laser-excited target from combining $2 \mathrm{D}$ radiative transport model with $1 \mathrm{D}$ hydrodynamic model at different times $\Delta \mathrm{t}$. b) Data points: Measured radius $r_{\text {dark }}(\Delta t)$ of darkened pump-excited plasma region at pump intensity $1.8 \times 10^{18} \mathrm{~W} / \mathrm{cm}^{2}$ (dark circles) and $3.7 \times 10^{17} \mathrm{~W} / \mathrm{cm}^{2}$ (triangles). Curves: Calculated radius $r_{c}(\Delta t)$ of critical surface at $\mathrm{z}=$ $\lambda_{\text {probe }} / 8$ at highest intensity (solid black), lower intensity (dotted) and neglecting radiative transport (dashed).

$1.8 \times 10^{18} \mathrm{~W} / \mathrm{cm}^{2}$, but assuming only Spitzer-Härm conductivity (i.e. puting $\kappa_{\mathrm{R}}=0$ ) is also shown (dashed curve). This curve falls well short of the data, thus clearly demonstrating the dominance of radiative transport.

The main discrepancy between measured $r_{\text {dark }}(\Delta t)$ and calculated $r_{c}(\Delta t)$ (including $\kappa_{R}$ ) is the faster initial evolution of the former. We believe that this is caused in part by the initially nonlocal character of the radiative transport, which cannot be described by a diffusion equation. This belief is based on an estimated Rosseland mean free path $\lambda_{\mathrm{R}} \sim 1 \mu \mathrm{m}$, comparable to the pump focal spot size, for our estimated initial conditions $\left(\mathrm{kT}_{\mathrm{e}} \sim 1 \mathrm{keV}, \mathrm{Z} \sim 10, \mathrm{n}_{\mathrm{e}} \sim 10^{24} \mathrm{~cm}^{-3}\right)$. Additional complication may be the 
dependence of $\lambda_{R}$ on material opacity, which has been greatly simplified in our model and the transport of hot electrons by $\mathbf{E} \times \mathbf{B}$ drift. [8,9] The latter effect pulls electrons out of the corona into vacuum, moves them radially away from the plasma region then drives them back into cool target material. These points can be addressed in future simulations using particle-in-cell (PIC) methods and future experiments using different target materials.

\section{REFERENCES}

1. A.J. Mackinnon et. al., "Effect of plasma scale length on multi-MeV proton production by intense laser pulses," Phys. Rev. Lett., 86, 1769 (2001).

2. A. Maksimchuk et. al., "Forward Ion Acceleration in Thin Films Driven by a High-Intensity Laser," Phys. Rev. Lett. 84, 4108 (2000).

3. T. Ditmire et al., "Supersonic ionization wave driven by radiation transport in a short-pulse laser-produced plasma," Phys. Rev. Lett. 77, 498 (1996).

4. O. Albert et al., "Generation of relativistic intensity pulses at a kilohertz repetition rate," Opt. Lett. 25,1125 (2000).

5. Y.B. Zel'dovich and Y.P. Raizer, Physics of Shock Waves and High-Temperature Hydrodynamic Phenomena, edited by W.D. Hayes and R.F. Probstein (Dover, New York, 2002)

6. W. L. Kruer, The Physics of Laser-Plasma Interactions (Addison-Wesley, 1988).

7. J.P. Christiansen et al., "MEDUSA a one-dimensional laser fusion code," Comp. Phys. Commun. 7, 271 (1974).

8. Y. Sentoku et. al., "Laser light and hot electron micro focusing using a conical target," Phys. Plasmas 11, 3083 (2004).

9. R. Kodama et. al., "Fast heating of ultrahigh-density plasma as a step towards laser fusion ignition." Nature 412, 798 (2001). 